


\title{
Le case di Lois Welzenbacher, dispositivi che reinventano lo spazio alpino
}

The houses of Lois Welzenbacher, devices that reinvent the Alpine space

\begin{abstract}
As an exponent of that "Tyrolean" generation of architects that in the Germanspeaking central-eastern Alps will be decisive in the specific declination of the themes of architectural modernity within the mountain context, Lois Welzenbacher realizes, between the end of the 1920s and the beginning of the 1930s, a series of houses and villas that reinvent the relationship between architecture and Alpine environment in completely new and inaugural terms. The Buchroithner house built in 1928-30 in Zell am See, the Rosenbauer house built in 1929-30 in Linz, and the Buchroithner house built in 1932 again in Zell am See establish a new way of relating to the space and to the Alpine landscape: they incorporate the mountain landscape, and at the same time they transform and change in relation to the topographic morphology of the site, giving life to an architecture that builds a relational dialectic with its surroundings in a completely new way. In this respect, the works of Lois Welzenbacher represent a decisive threshold in the conceptualization of the construction in the Alps.
\end{abstract}

\section{Antonio De Rossi}

Architect, PhD, full professor of Architectural and Urban Design at the Politecnico di Torino and director of the research centre «Istituto di Architettura Montana» (IAM). Between 2005 and 2014 he was vice director of «Urban Center Metropolitano" of Turin. He's author of various architectural projects in the Alps. He published the work in two volumes La costruzione delle Alpi (Donzelli 2014 and 2016) that have won the "Rigoni Stern" prize and the "Acqui Storia» prize and the book Riabitare l'Italia. Le aree interne tra abbandoni e riconquiste (Donzelli, 2018).

\section{Laura Mascino}

Laura Mascino, architect and $\mathrm{PhD}$, is professor of Urban Planning at the Politecnico di Milano. She works at the Istituzione Veneziana, where she deals with social housing and welfare. She has won several national and international competitions, and has carried out architectural projects in Italy, Great Britain, Japan. Recently, she coordinated several urban and territorial regeneration projects: Terraferma - Venetian Agricultural Park, DD Social in Venice Dorsoduro and Crocevia Piave in Mestre. She is among the authors of the book Riabitare l'Italia. Le aree interne tra abbandoni $e$ riconquiste (Donzelli, 2018).

Keywords

Tyrol, modernism, design, landscape, panorama. 
Esponente di punta di quella generazione di progettisti che, come scrive Bettina Schlorhaufer in questo numero di «ArchAlp», inventano il "Tyrolean Modernism", Lois Welzenbacher, nato a Monaco di Baviera nel 1889, è certamente uno degli autori che con le sue costruzioni ha saputo definire un rapporto specifico e originale tra architettura moderna e ambiente alpino. Al pari di Carlo Mollino, i progetti di Welzenbacher - come ha ricordato Bruno Reichlin nel fondamentale saggio Quando gli architetti moderni costruiscono in montagna (1996) - stabiliscono una relazione dialettica e dinamica tra architettura e montagna che non si limita alla trasposizione di viste panoramiche dentro l'edificio, ma che attraverso l'interiorizzazione dello spazio alpino giunge a modificare la costruzione al fine di accogliere, come direbbero i francesi, la montagnité dell'ambiente come materiale e componente primaria del fare progettuale.
All'invenzione della monofalda tra la fine degli anni Venti e l'inizio dei Trenta come peculiare dispositivo per mettere in relazione la costruzione con l'ambiente alpino - si pensi allo Sporthotel Monte Pana realizzato da Franz Baumann nel 1930-31 -, si affiancano le ricerche e le sperimentazioni architettoniche di Lois Welzenbacher. Nel periodo a cavallo tra gli anni Venti e Trenta l'architetto realizza infatti una serie di case - ma analoghe considerazioni potrebbero essere fatte osservando le strategie da lui perseguite nel Terrassenhotel realizzato nel 19321934 a Oberjoch - che reinventano profondamente la relazione tra architettura e paesaggio montano, giungendo a una sorta di messa in forma e di estrusione della materia solida dell'architettura a partire dalla prefigurazione di un sistema di viste e di percorsi preludenti le vedute panoramiche.

Un tema per molti versi di discontinuità e di rottura, se si pensa ai modi con cui l'architettura realiz-

Casa Buchroithner,

Zell am See. Vista esterna dell'ingresso

(nord) (Harbers,

1931).

Fig. 1

Casa Buchroithner,

Zell am See, vista sud (Harbers, 1931).

Fig. 2

Casa Buchroithner, Zell am See, vista sud.

Tutte le successive immagini di casa

Buchroithner sono tratte da Sarnitz,

1989.

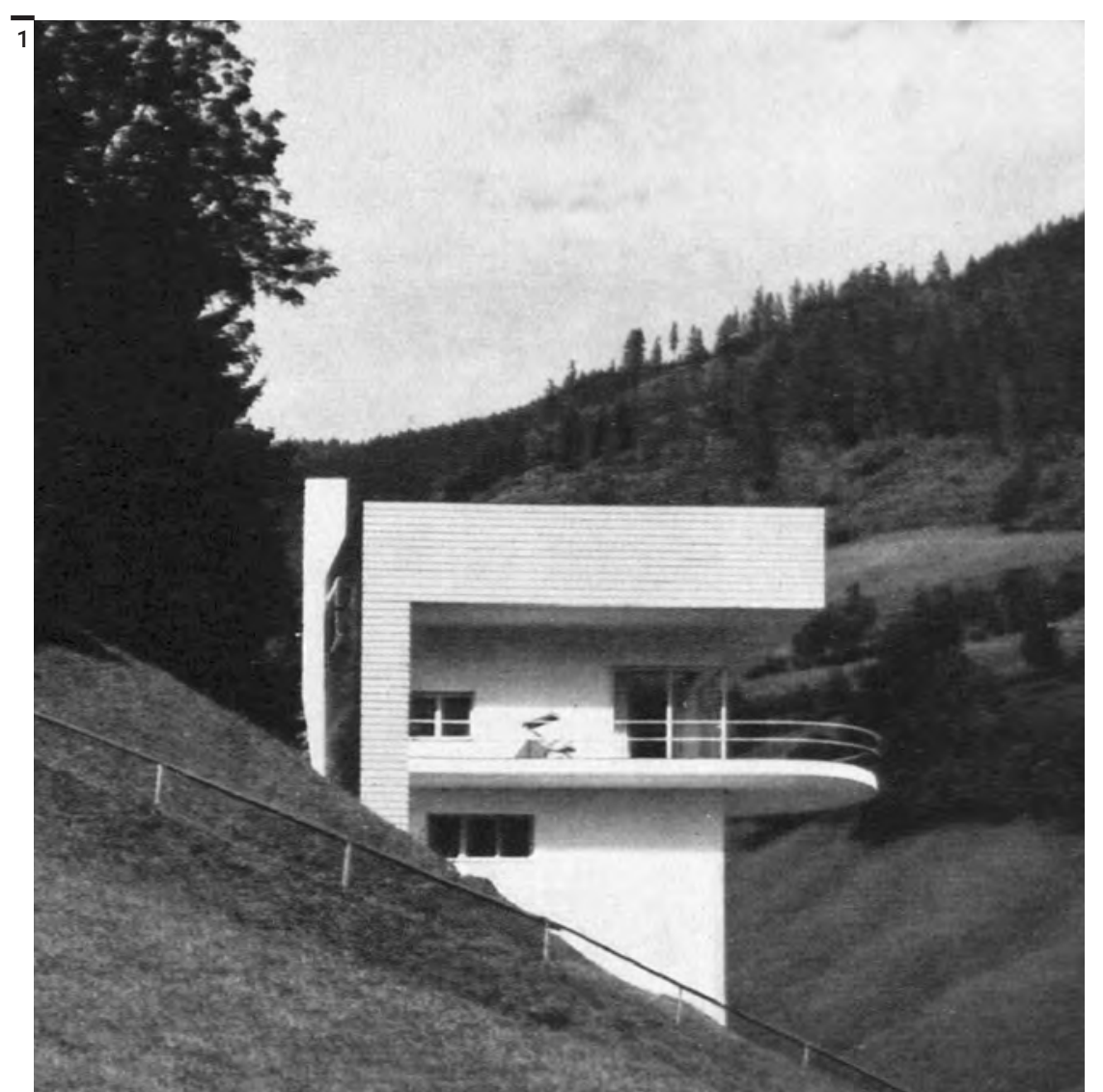

$\overline{2}$

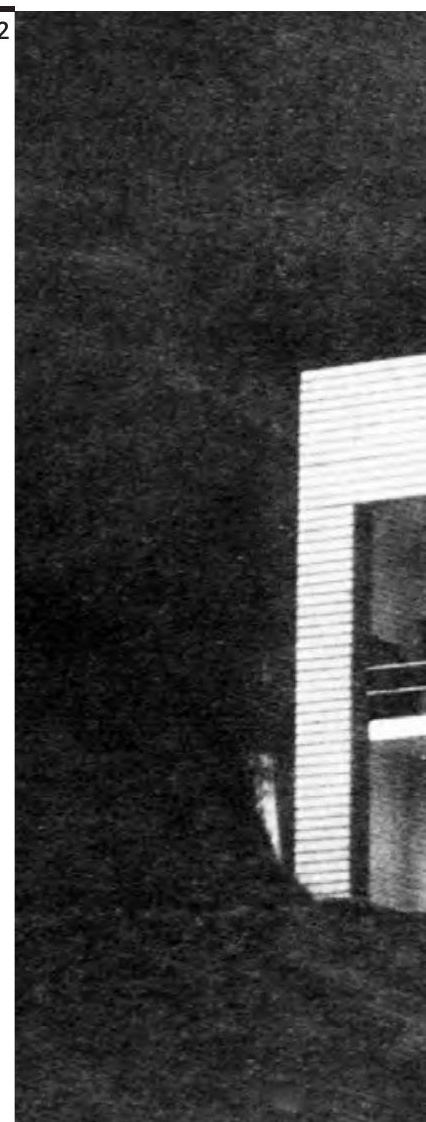



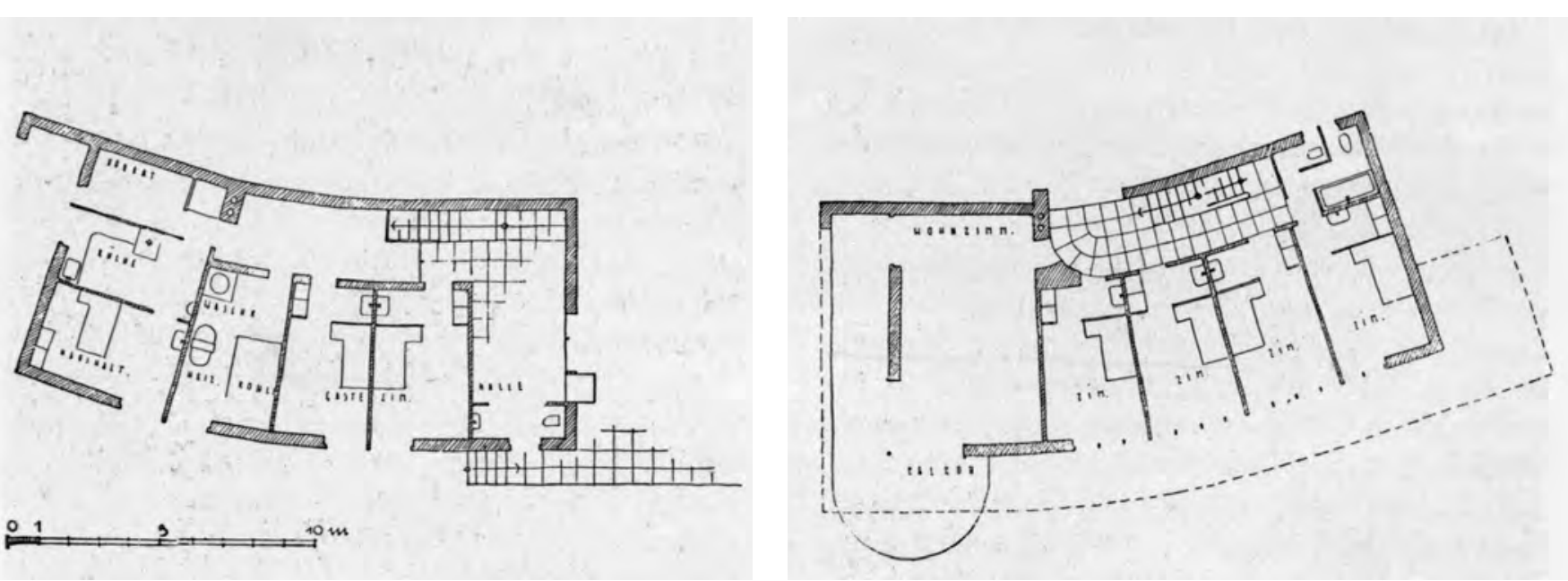

zata in montagna aveva fino a quel momento genericamente affrontato la questione, e che evidenzia una mutazione e uno spostamento concettuale rilevante. L'ambiente alpino sembra infatti progressivamente perdere, nell'interpretazione degli archiFig. 3 tetti moderni operanti in montagna in quegli anni, Pianta del piano le valenze di paesaggio culturale, per come si erano terra. stratificate nel corso dell'Ottocento, per trasformarsi in spazio innanzitutto topografico e panoramico Fig. 4 riassumibile in pochi tratti essenziali.

Pianta del piano primo.
Gli edifici della fine anni Venti e inizio anni Trenta sono anticipati dal progetto di casa Settari, realizza-

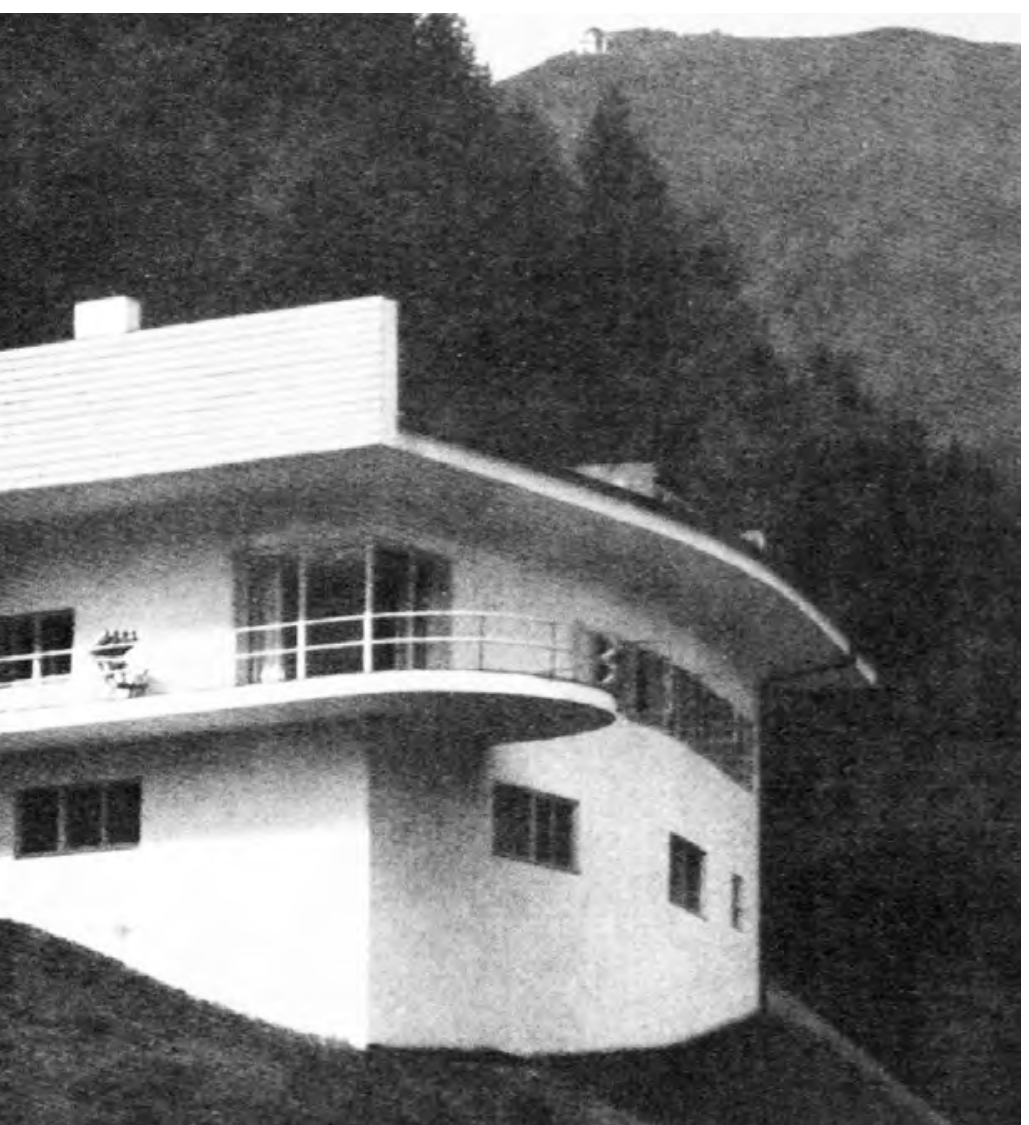

ta da Welzenbacher nel 1922-23 a Dreikirchen-Tre Chiese, in val d'Isarco in Alto Adige, su un leggero ripiano di un lungo versante. Un'architettura che, al di là delle sembianze esteriori apparentemente tradizionali, viene a costruirsi intorno al doppio tema dell'ascensione lungo una spirale e della definizione di una sequenza di viste obbligate sul panorama alpino circostante. Da questo punto di vista il progetto per casa Settari risponde a istanze che sono centrali nella visione di Welzenbacher: la capacità di cogliere le configurazioni in potenza esistenti in un sito in termini di valorizzazione del rapporto visivo e topografico tra costruzione e paesaggio, e la conseguente messa a punto di un dispositivo spaziale e architettonico in grado di massimizzare questa interazione.

Un dispositivo di natura essenzialmente dinamica: a partire dall'ambiente esterno, la persona viene guidata dentro l'edificio, dove tramite scale e percorsi, viste parziali e sempre mutevoli dell'intorno, viene infine condotta a una sorta di visione finale del paesaggio complessivo del luogo. Questa macchina che produce viste funziona quindi come una sorta di doppio imbuto - che prima accoglie, poi accompagna e comprime, per aprirsi infine sul panorama, esattamente come avverrà qualche anno dopo con i progetti di Carlo Mollino - finalizzato al potenziamento e all'intensificazione dell'esperienza percettiva, visiva, materica della montagna.

La casa Buchroithner, realizzata da Welzenbacher nel 1928-30 sopra Zell am See, è sotto questo profilo emblematica: un percorso attraverso gli spazi interni dell'edificio che sembra procedere parallelamente al movimento della veletta aggettante che attraversa orizzontalmente tutta la facciata convessa, e che trova compimento nell'affaccio-sbalzo sul lago. Evento culmine e conclusivo della sequenza che viene sottolineato dalla soletta che si piega di 
$90^{\circ}$ - quasi a trasformarsi in cornice e boccascena moderna a cui è affidato il compito dell'interazione tra architettura e paesaggio -, dall'apertura che squarcia l'angolo pieno della costruzione, e dall'andamento perpendicolare, solo apparentemente contraddittorio, della terrazza con terminazione semicircolare.

Se la casa Rosenbauer, costruita a Linz nel 1929-30, si configura - pur rappresentando un'icona dell'architettura moderna - essenzialmente come la solidificazione quasi mendelsohniana di un punto di vista panoramico, la casa Heyrovsky ripropone i medesimi sofisticati procedimenti osservati nella casa Buchroithner. L'edificio, realizzato nel 1932 sempre a Zell am See, affronta nuovamente la dialettica tra architettura e paesaggio del lago. L'operazione di sovrapposizione e di leggero slittamento dei due volumi in curva del piano terreno e del primo piano - resa possibile dalla chiusura della loggia sul lato sinistro, che trasforma il gioco di piani in incastro di scatole - diventa l'elemento base su cui costruire uno sventagliamento di scorci e vedute panoramiche. In questo processo di panoramizzazione - e qui sta uno dei tratti decisivi dell'opera di Welzenbacher - il paesaggio subisce una sorta di mutazione metasemica. Da elemento esterno, che preesiste e vive indipendentemente dal progetto, esso diventa materiale interno al progetto, esito stesso dell'azione progettua-

Fig. 5

Casa Heyrovsky, Zell am See. Pianta del piano terra.

Tutte le immagini di casa Heyrovsky sono tratte da Sarnitz, 1989.

Fig. 6

Pianta del piano primo.

Fig. 7

Fotografia del lato est con vista sul lago.

Fig. 8

Vista da sud-ovest.

Fig. 9

Casa Rosenbauer a Linz. Vista del lato sud sul soggiorno e sulla camera da letto. Tutte le immagini di

casa Rosenbauer sono tratte da Harbers, 1931. le. Divenendo un costrutto del progetto, il paesaggio - secondo una procedura cara ai Moderni, e che nelle Alpi acquisisce particolare visibilità e rilevanza - tende quindi a trasformarsi in natura, in sfondo astratto e topografia geometrica, sfuggendo alle connotazioni culturali insite nel termine.

Le macchine panoramiche messe a punto da Lois Welzenbacher sanciranno in maniera definitiva un'idea di architettura alpina in cui la costruzione non solo definisce visuali e affacci sul panorama, ma prende forma proprio nell'interazione col paesaggio. Da questo punto di vista sono molti i progetti degli anni trenta che iniziano a fare proprio questo tema. A titolo esemplificativo - e non a caso si tratta di architetture tutte oggetto di questo numero di «ArchAlp» - si può citare la villa Borsotti a Balme, nelle valli di Lanzo, che nei primi anni del decennio vede la collaborazione dell'artista Gigi Chessa con l'architetto Umberto Cuzzi. Qui, lungo un tracciato leggermente incurvato che apre alla visione dell'alta valle da punti di vista differenti, un volume in legno a copertura piana viene ad appoggiarsi in aggetto su un basamento in pietra, lo sbalzo sostenuto da esili colonnine in cemento che generano uno stretto porticato: l'effetto del potente colpo d'ombra fa librare la parte sovrastante. Oppure la villa La Roccia a Canobbio di Luigi Vietti, che in linea con le sperimentazioni di Wel- zenbacher costruisce su un impianto planimetrico irregolarmente circolare un sistema di visuali a ventaglio sulle acque del Lago Maggiore e sui rilievi che lo circondano.

L'importanza della rottura operata da Lois Welzenbacher può essere osservata per contrasto nel modo con cui figure di provata fede funzionalista, come ad esempio Alberto Sartoris, affrontano medesimi temi: la casa Morand-Pasteur da lui costruita nel 1934-35 a Saillon nel Vallese resta estranea al paesaggio dei vigneti e delle cime che circondano il grande asse vallivo, configurandosi come un oggetto stereometrico e assoluto che viene a generarsi esclusivamente a partire dalle proprie logiche e codici interni, e lo stesso si può dire per la chiesa di Lourtier sempre nel Vallese di qualche anno precedente.

Questo tema di un'architettura che «fa un gesto» (Reichlin, 1996: 104), che non solo incornicia visioni panoramiche, ma che viene letteralmente a modellarsi nell'interazione visiva col paesaggio e

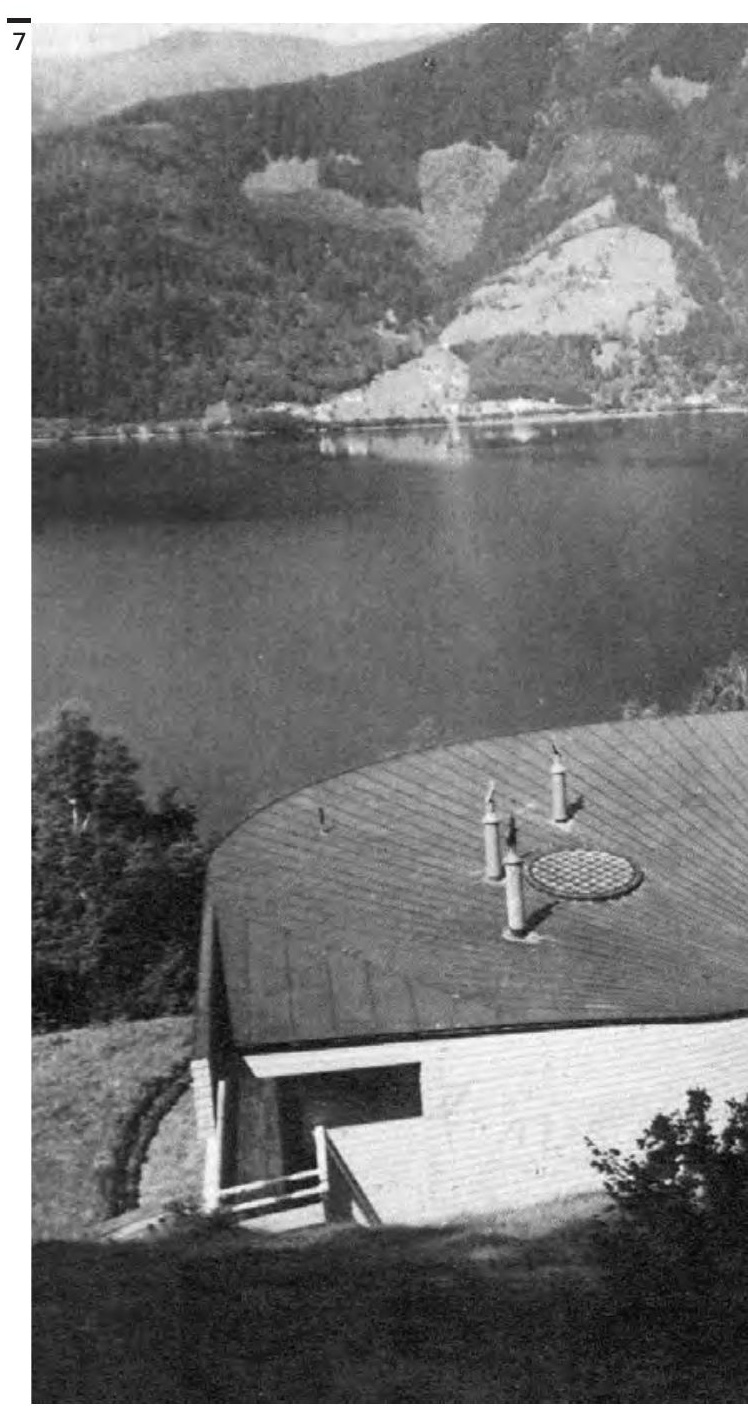



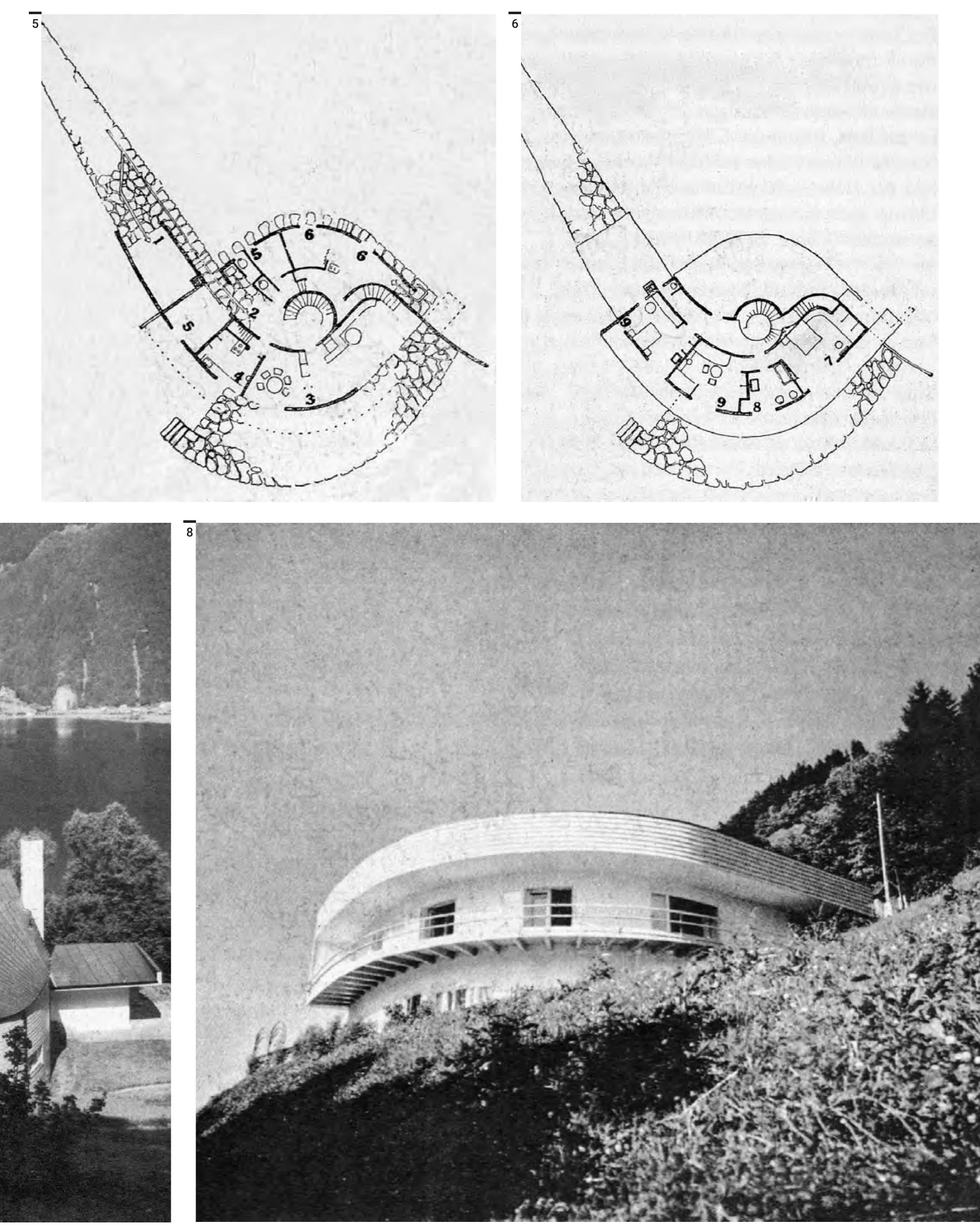


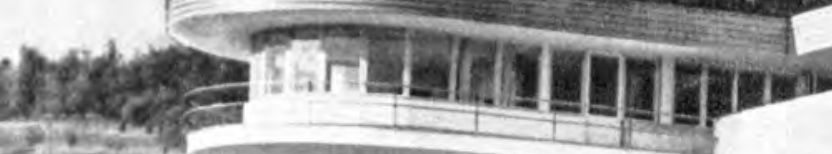
$+102+3 x+3$

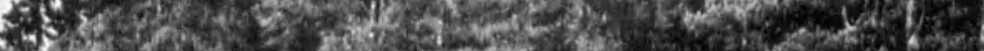

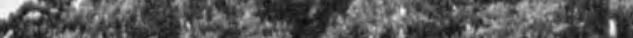

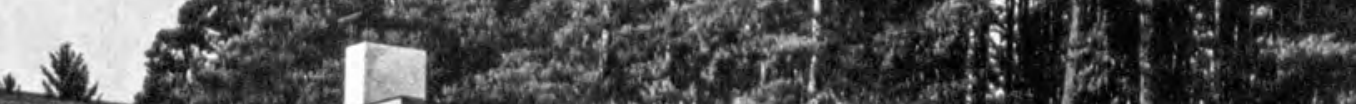
T.2.

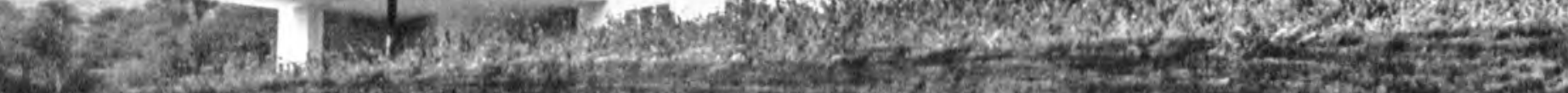

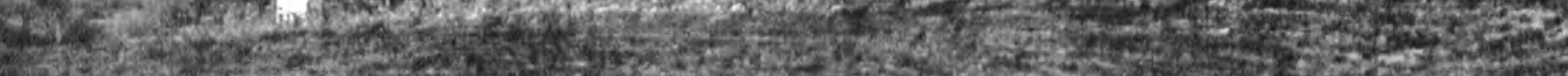

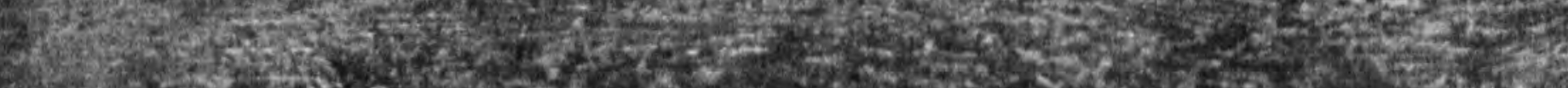

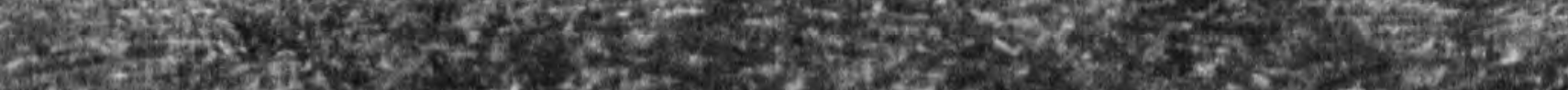

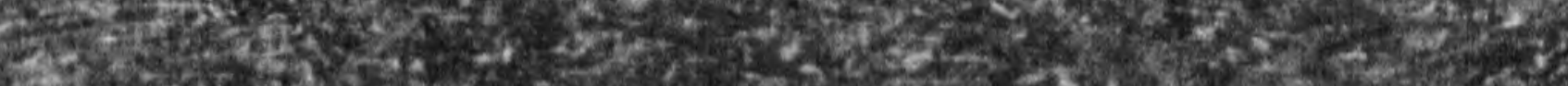

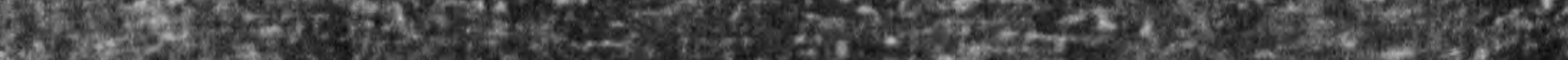
a.t.

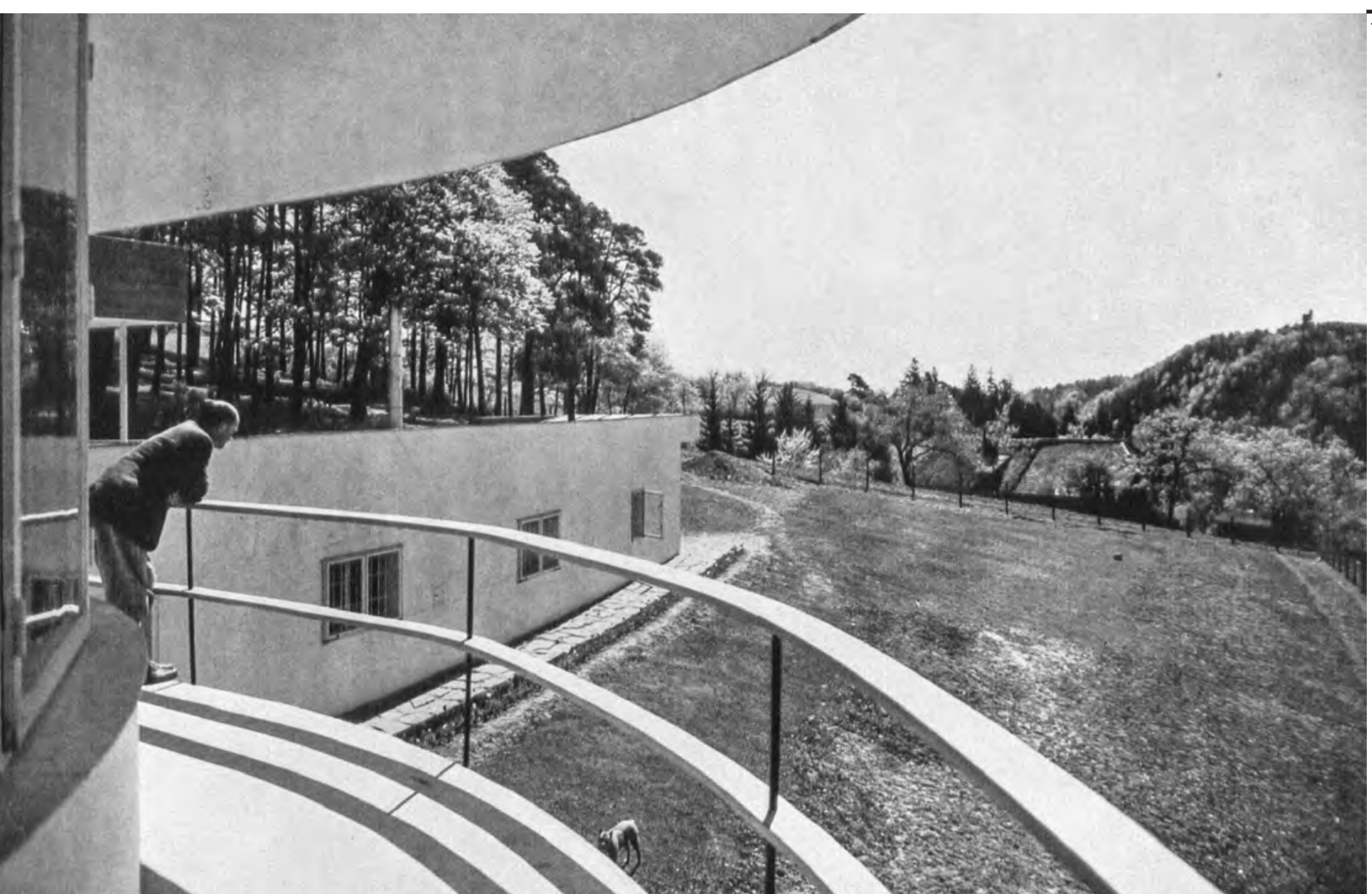




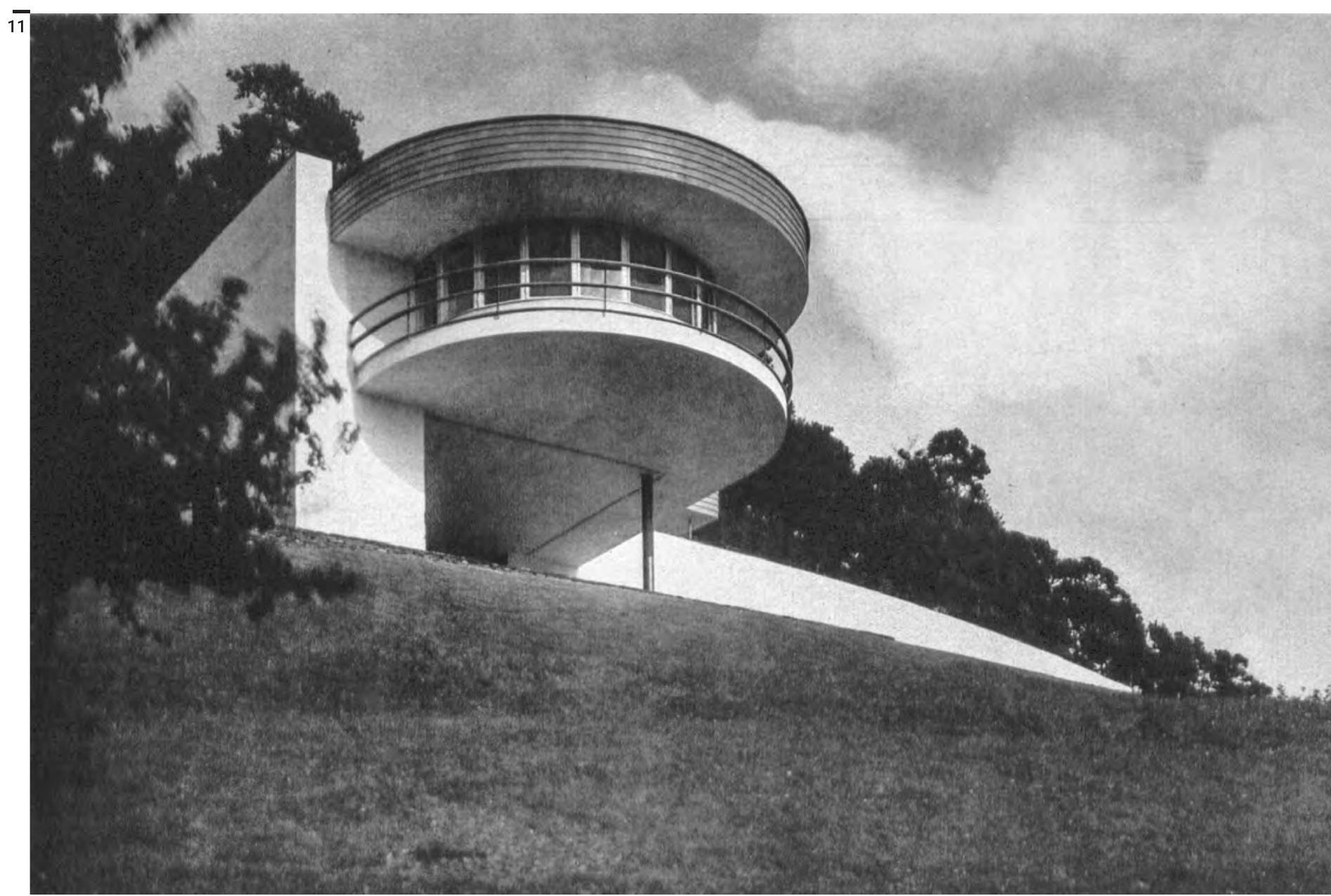

Fig. 10

Vista dalla veranda

a sud.

Fig. 11

Vista esterna sul soggiorno, lato sud-ovest. tramite l'interpretazione costruttiva della spazialità fisica della montagna, verrà affrontato da Maurice Braillard nella straordinaria stazione di arrivo della funivia di Salève, realizzata sopra il Lago Lemano nel 1932, e conoscerà ulteriore sviluppo nei progetti messi a punto da Carlo Mollino negli anni Quaranta e Cinquanta. Proprio il riferimento all'architetto torinese permette di ricordare un progetto dell'architetto di Basilea Paul Artaria (1936: 118-119), che nel 1932 disegna, senza realizzarla, una Wochenendhaus a Wartenberg in alta Bavie- ra, che prefigura in modo praticamente inaugurale temi che ricorreranno incessantemente nell'opera di Carlo Mollino, venendo infine sintetizzati nella casa dell'altopiano di Agra del 1952-54: un edificio a sviluppo longitudinale su pendio, costruito intorno a un'ossatura lignea, che battendo orizzontalmente la quota viene a configurarsi verso valle come un potente aggetto, concludendosi con una terrazza dischiusa verso il paesaggio. È il definitivo compimento di quanto inaugurato e aperto dalle opere di Lois Welzenbacher.

\section{Bibliografia}

Achleitner Friedrich, Uhl Ottokar (1968), Lois Welzenbacher 1889-1955, Residenz Verlag, Salzburg Artaria Paul (1936), Schweizer Holzhäuser, Wepf, Basel.

Harbers Guido (1931), Lois Welzenbacher. Arbeiten der Jahre 1919 bis 1931, Georg D.W. Callwey, München.

Hauser Sigrid (1990), Idee, Skizze, Foto. Zu Werk und Arbeitsweise Lois Welzenbacher, Löcker, Wien.

Reichlin Bruno (1996), «Die Moderne baut in den Bergen - Quando gli architetti moderni costruiscono in montagna», in Mayr Fingerle Christoph (a cura di), Neues Bauen in den Alpen - Architettura contemporanea alpina. Architekturpreis - Premio d'architettura 1995, Birkhäuser, Basel-Boston-Berlin.

Sarnitz August (1989), Lois Welzenbacher. Architekt, 1889-1955, Residenz Verlag, Salzburg-Wien. 